\title{
Familial Dominant Drusen with Cnvm: A Case Report
}

\author{
Authors \\ Dr Anusha Pawar, Dr Sonal Bangwal, Dr Piyush Bajaj, Dr Tripti Choudhary \\ Venu Eye Institute and Research Centre \\ 1/31, Sheikh Sarai Institutional Area- 2, New Delhi India 110017 \\ Corresponding Author \\ Dr Anusha Pawar \\ Se-161 Shastri Nagar, Ghaziabad, Uttar Pradesh India 201002 \\ 09953927339, 08383949510, Email: anushapawar120987@gmail.com
}

\begin{abstract}
Hereditary drusen are an autosomal dominant entity. The most important factor is age-related so-called typical drusen. But this entity is characterized by an early appearance of drusen. The clinical appearance of familial drusen is variable, ranging from a few large, coarse lesions to numerous tiny dots. Because of the possibility of loss of vision in the younger years, an early diagnosis of familial drusen in necessary. No known effective treatment exists for Familial Dominant Drusen. Follow up is required in cases of Familial Dominant Drusen as occurence of CNVM is a possibility. Prompt treatment is required for the same.

KEYWORDS: Familial dominant drusen, choroidalneovascular membrane, malattialeventinese, Doyne honeycomb retinal dystrophy.
\end{abstract}

\section{INTRODUCTION}

Hereditary drusen are an autosomal dominant entity. The most important factor is age-related socalled typical drusen. Apart from the genetics and family history, characterized by the earlier appearance of drusen (at 20 to 30 years of age) ${ }^{1}$, drusen present in the nasal half of the fundus and earlier reduction in vision. Because of the possibility of loss of vision in the younger years, an early diagnosis of familial drusen in necessary. ${ }^{2}$ We report a case of familial dominant drusen with choroidalneovascular membrane which presented to us.

\section{CASE REPORT}

A 56 year old man presented to our OPD with the chief complaint of diminution of vision in either eye since 1 year. Diminution of vision was insidious in onset, painless and gradually progressive in nature. No significant ocular history. Family history was negative. His visual acuity was Finger Counting $4 \mathrm{~m}$ in right eye and Finger Counting $5 \mathrm{~m}$ in LE. Anterior segment was normal in both the eyes. Pupillary reaction and color vision was normal in both eyes. IOP was within normal limits. He had nuclear grade 1 cataract in both eyes. Posterior segment showed multiple drusens over posterior pole along with scarring at macula in both the eyes which was more in the left eye (Figure 1 and Figure 2). FFA and OCT macula revealed CNVM with scarring in both eyes. (Figure 3 and Figure 4). On the basis of clinical findings and investigations a diagnosis of Familial Dominant Drusen with Choroidal 


\section{JMSCR Vol||05||Issue||08||Page 26300-26302||August}

Neovascular Membrane in both the eyes was made. No known effective treatment exists for Familial Dominant Drusen. Follow up is required in cases of Familial Dominant Drusen as occurence of CNVM is a possibility. Prompt treatment is required for the same. Our case had healed CNVM scars so the vision loss is permanent and requires a regular follow up to detect further deterioration of vision.

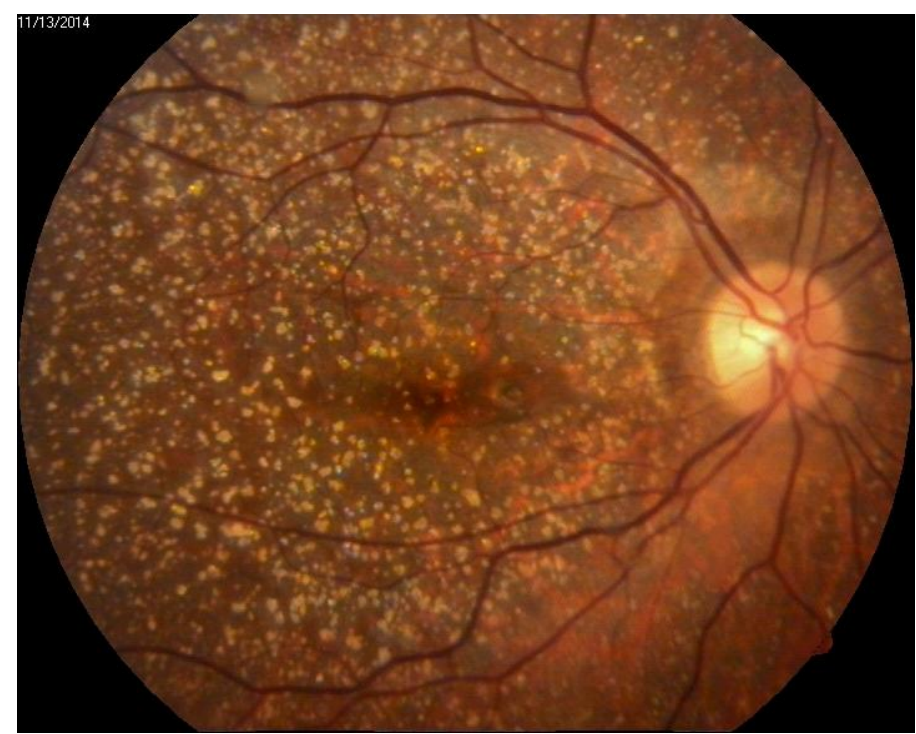

Figure 1: Familial Dominant Drusen Right Eye

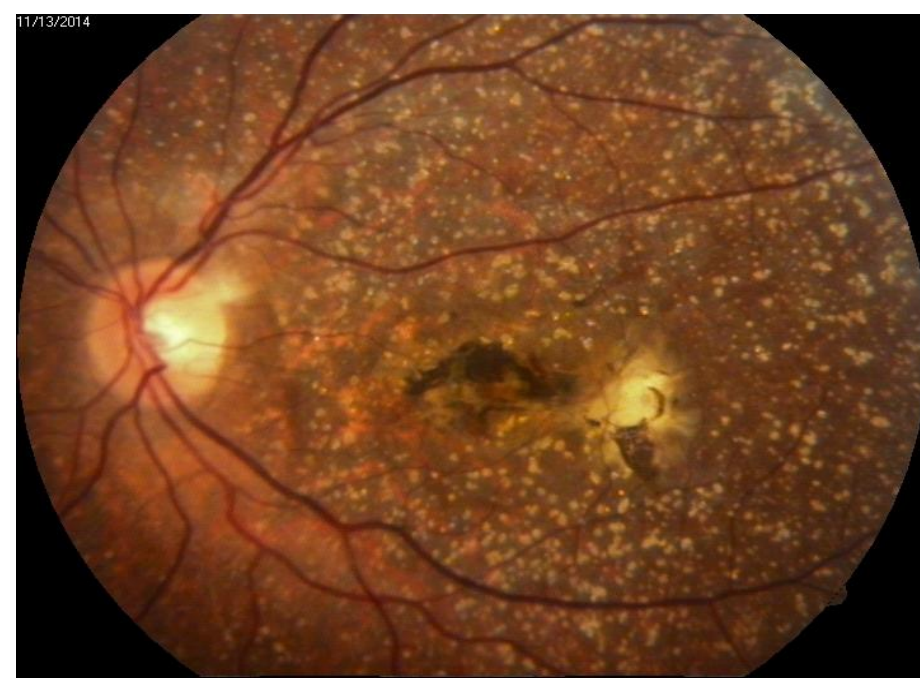

Figure 2: Familial Dominant Drusen with Cnvm Left Eye

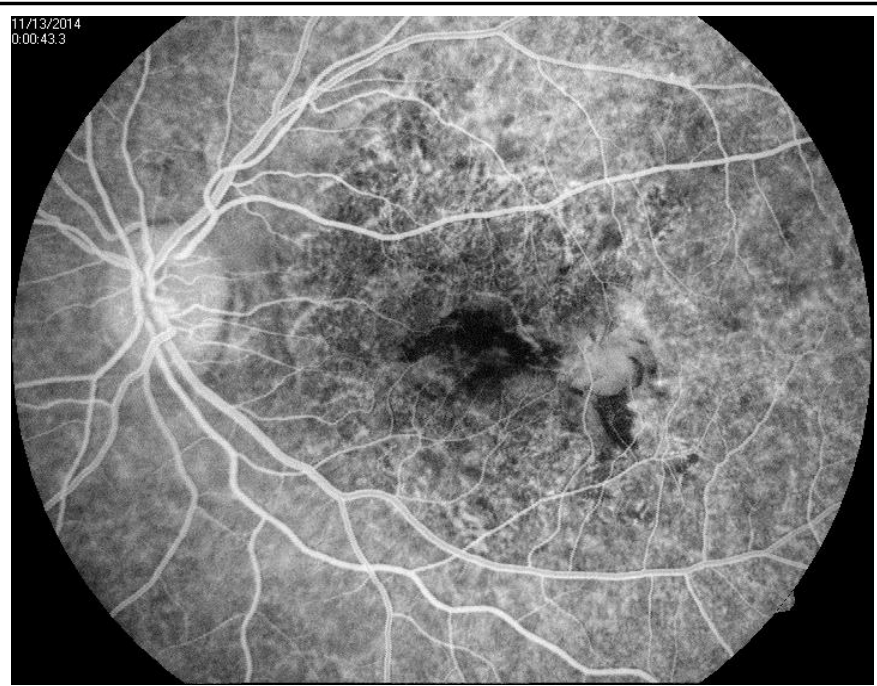

Figure 3: Ffa Right Eye

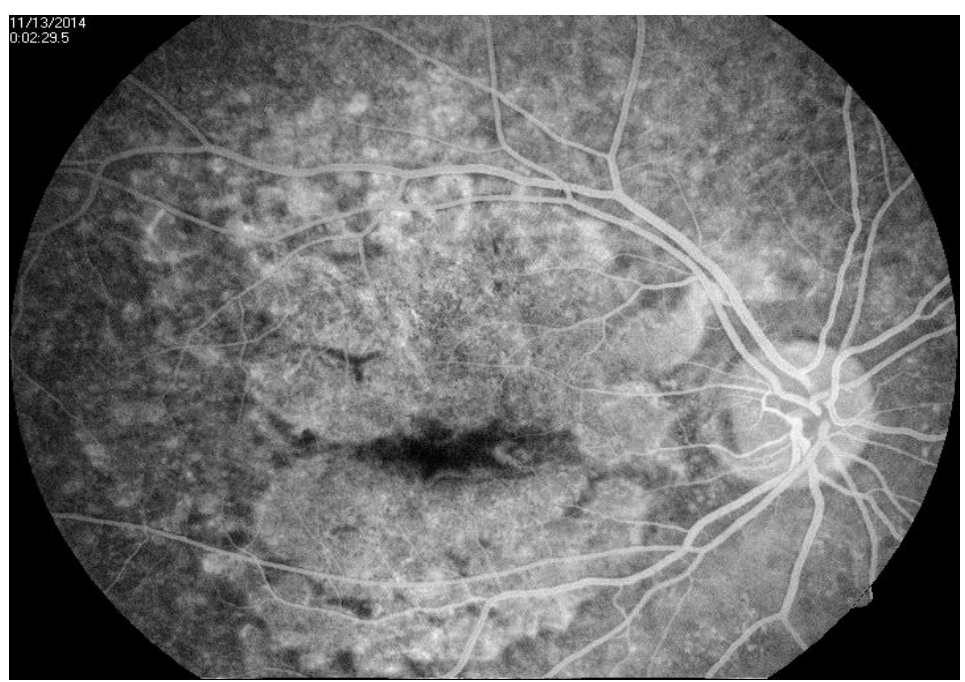

Figure 4: FFA Left Eye

\section{DISCUSSION}

Malattia Leventinese, also known as dominant radial drusen or Doyne honeycomb retinal dystrophy, was first described in patients living in the Leventine Valley in canton Ticino of southern Switzerland in 1925. A missense mutation (Arg345Trp) in the gene EFEMP1 on chromosome 2 was discovered to be causative for both conditions. This gene codes for an epidermal growth factor (EGF)-containing, fibrillin-like, extracellular matrix protein. ${ }^{2}$ The phenotype is distinctive because the drusen develop in a radiating pattern from the fovea. Malattia Leventinese is reported to be autosomal dominant with variable expressivity phenotype. Characteristic clinical findings consist of radial macular drusen, large confluent drusen, and 
juxtapapillary drusen, which can be an isolated finding. Early visual symptoms, typically starting at age of 30-50 years, include reduced central vision, photophobia, and metamorphopsia. The vision gradually deteriorates over many years. No curative treatment is available for Malattia Leventinese; however, some prophylactic argon laser treatment has been promising in improving visual acuity and reducing the drusen volume. Anti-vascular endothelial growth (VEGF) treatment is efficient in stabilizing a choroidalneovascular membrane. ${ }^{3}$ The clinical appearance of familial drusen is variable, ranging from a few large, coarse lesions to numerous tiny dots sometimes called basal laminar or cuticular drusen. The cuticular drusen phenotype is highly associated with the Tyr402His variant of the complement factor $\mathrm{H}(\mathrm{CFH})$ gene. Fluorescein angiography often shows more extensive drusen and RPE changes than are evident on ophthalmoscopy. The ERG and EOG are typically normal. Central vision is good as long as the drusen are discrete and extra foveal. However, these patients may be at a greater than normal risk for macular degeneration as they age. ${ }^{2}$ Because of the possibility of loss of vision in the younger years, an early diagnosis of familial drusen in necessary to be able to perform thorough family and job counseling. ${ }^{1}$ Differential Diagnoses for Malattia Leventinese is Stargardt disease (fundus flavimaculatus), Sorsby macular dystrophy, Pattern dystrophies, Zermatt macular dystrophy, Best disease (in its later stages). ${ }^{4}$

\section{ACKNOWLEDGEMENTS: None SOURCE OF FUNDING: Nil}

\section{REFERENCES}

1. Kasmann B, Volcker H. Familial drusen of the macula. Fortschr Ophthalmology. 1990;87(6):567-70.

2. [Internet]. ONE NETWORK. 2017 [cited 1 August 2017]. Available from:
http://file:///C:/Users/hp/Desktop/Familial \%20(Dominant)\%20Drusen.html

3. Vaclavik V, Munier F. Macular Dystrophies. 2017.

4. Eye Rounds.org: Malattia Leventinese (Familial Dominant Drusen): 30 year-old female with drusen [Internet]. Webeye.ophth.uiowa.edu. 2017 [cited 1 August 2017]. Available from: http://webeye.ophth.uiowa.edu/eyeforum/c ases/48-Malattia-Leventinese-FamilialDominant-Drusen.htm 